\title{
Author index to Volume 3
}

(BR) Book review; (D) Digest; (E) Editorial; (L) Letter; (O) Opinion; (R) Research; (S) Summary; (T) Toolbox

Bader J 91 (E), 105 (S)

Bebermeyer RD 39 (S)

Benn DK 62 (E)

Benson P $86(\mathrm{~L})$

Brothag D $52(\mathrm{~S})$

Bruce M 76 (S)

Carpio L 11 (S)

Chen L 5 (R)

Cleaton-Jones P 75 (S)

Cohen R 51 (S)

Conway S 5 (R)

Coulthard P 107 (S)

Creugers N 77 (S)

Dodson TB 23 (T), 100 (S)

Farsai PS 79 (S)

Garcia R 17 (D)

Hausen HW 41 (S)

Henshaw M 79 (S)

Ismail AI 10 (S)
John JH 16 (S), 40 (S), 50 (S), 111 (BR)

Jokstad A 2 (E), 89 (E), 96 (S)

Juniper R 69 (S), 70 (S)

Kalha A 86 (L)

Kleijnen J 32 (E)

Kreulen C 102 (S)

Lawrence A 27 (T)

Li Y 47 (S)

Lumley P 72 (S)

Mansfield P 32 (E)

Matthews DC 12 (S), 103 (S)

Mattila K 30 (L)

McGrath C 73 (S)

Meechan JG 67 (S)

Mettes DG $81(\mathrm{~T})$

Mew J 35 (O)

Murzyn L 5 (R)

Needleman I 14 (S)

Niederman R 5 (R), 17 (D), 30 (L)

Nyvad B 53 (S)
O’Mullane D 43 (S)

Pitts N 93 (E)

Quant T 60 (L)

Randall RC 85 (L)

Richards D 1 (E), 27 (T), 31 (E), 32 (E), $37(\mathrm{~S}), 54(\mathrm{D}), 56(\mathrm{~T}), 60(\mathrm{~L}), 61(\mathrm{E})$, 80 (S), 84 (BR), 109 (T)

Sheiham A $22(\mathrm{~T})$

Shugars D 91 (E)

Sohn W 10 (S)

Tavender E 20 (D)

Thomas D 45 (S), 49 (S)

van der Sanden WJM $81(\mathrm{~T})$

Vrijhoef TMA 85 (L)

Weintraub JA 64 (S)

Wilson NHF 85 (L)

Yeo JF $66(S)$ 